\title{
Cystine Stone Formers Have Impaired Health-Related Quality of Life Compared with Noncystine Stone Formers: A Case-Referent Study Piloting the Wisconsin Stone Quality of Life Questionnaire Among Patients with Cystine Stones
}

\author{
Necole M. Streeper, MD, Margaret L. Wertheim, MS, Stephen Y. Nakada, MD, and Kristina L. Penniston, PhD, RD
}

\begin{abstract}
Introduction: Cystinuria is a rare cause of urolithiasis. Affected patients have an earlier onset and more aggressive disease than patients with other stone etiologies. We assessed the health-related quality of life (HRQOL) of cystine stone-forming patients using the disease-specific Wisconsin Stone Quality of Life questionnaire (WISQOL).

Methods: Cystine patients treated in our stone clinics $(n=12)$ completed the WISQOL; information about medical and stone histories was gathered. Patients were matched with noncystine stone formers $(n=12)$ for gender, age, and comorbidities. In addition, a second control group $(n=90)$, also from our institution and consisting of mixed calcium stone formers, was included. WISQOL responses were compared between groups. Results: Cystine patients had significantly lower total WISQOL scores than noncystine patients. Compared with noncystine stone formers, cystine stone formers also had lower HRQOL scores for subscales (domains) related to social impact, emotional impact, disease impact, and vitality ( $p \leq 0.04$ for all). On specific items, cystine patients reported significantly more sleep problems $(p=0.02)$, more bother with nocturia $(p=0.03)$, and feeling tired or fatigued $(p=0.02)$. Among those with current stones, cystine patients scored lower than noncystine patients for total score and in two of four domains.

Conclusions: Using a stone-specific questionnaire, patients with cystine stones have lower HRQOL compared with noncystine stone formers. Identifying and addressing specific areas of decrement in patients with cystine stones may improve disease management and patients' HRQOL.
\end{abstract}

Keywords: cystine, cystinuria, health-related quality of life, kidney stones, quality of life

\section{Introduction}

C YSTINURIA IS AN INBORN error of metabolism (either autosomal recessive or autosomal dominant with incomplete penetrance) characterized by defective kidney transport of cysteine and dibasic amino acids. It was one of the first inborn errors of metabolism identified in humans. ${ }^{1}$ Cystine stones are formed secondary to a urinary cystine concentration above solubility $(\sim 250 \mathrm{mg} / \mathrm{L}))^{2,3}$ Patients with cystinuria have an early age of onset; the first symptomatic stone typically presents within the first two decades of life. ${ }^{4}$ There is no cure for cystinuria, and patients with the disorder typically form stones frequently and undergo multiple surgical procedures over time. Renal obstruction and staghorn calculi are not uncommon.

The goals of therapy are centered on reduced cystine stone formation. Goals include: (1) increasing cystine solubility with urinary alkalinization, (2) solubilizing insoluble cystine to cysteine with cystine-binding thiol medications, (3) decreasing the urinary saturation of cystine with higher urine output, and (4) reducing urinary cystine excretion by maintaining a low sodium diet and, if not contraindicated, a diet lower for the essential amino acids methionine and cysteine. While these strategies either alone or in combination may be effective in reducing cystine stone recurrence, some patients have difficulty adhering to their prescribed prevention regimen $^{5}$ and are therefore at even higher risk for stone recurrence and impaired renal function. ${ }^{6-9}$

Urolithiasis of any type can be chronic and confers complex challenges and symptoms upon patients, including decrements in quality of life and specifically health-related quality of life (HRQOL). The recurrence of stones is dependent, in part, on patients' compliance with directed medical and dietary therapies. When multiple strategies are used,

Department of Urology, University of Wisconsin School of Medicine and Public Health, Madison, Wisconsin. 
which may include both pharmacologic and dietary, the prevention of stones can be burdensome to patients. HRQOL is thus an important end point in managing patients with recurrent stones not only to document the impact of stones themselves on patients' HRQOL but also the impact of therapy. This may be especially true for patients with cystinuria as their stone recurrence rates and need for surgical interventions are higher. ${ }^{3}$ Two previous studies which used the SF-36 questionnaire (a general HRQOL survey) to assess the HRQOL of cystine stone formers reported significant effects on the HRQOL of cystine stone formers both compared with the general public ${ }^{10}$ and to noncystine stone formers. ${ }^{11}$ There has not previously been a study evaluating the HRQOL of patients who form cystine stones using a disease-specific HRQOL survey.

Disease-specific questionnaires are known to be superior to general HRQOL measures, especially for conditions with a wide range of symptoms or variable expression between patients. Disease-specific questionnaires can more deeply probe unique disease effects. ${ }^{12}$ Identifying specific areas of lower HRQOL may assist in improving disease management strategies (Fig. 1). The objective of this study was to compare the HRQOL of patients with cystinuria to patients with other stone-forming etiologies using the disease-specific Wisconsin Stone Quality of Life questionnaire (WISQOL). ${ }^{13}$

\section{Patients and Methods}

After obtaining approval from our institutional review board, we identified 35 patients with cystinuria from our stone clinics. Of these, 21 had been seen within the past 5 years. After extending the invitation to participate, 13 patients consented and were enrolled (1 patient was excluded from analysis due to missing medical information). Patients from our clinic with a history of noncystine stones were sequentially reviewed from most recent clinic appointments and matched to patients in the cystine group according to gender, age (within 4 years), comorbidities, and whether or not they were a recurrent (i.e., $>1$ event) stone former. These matched noncystine stone-forming patients were used as referents to control for effects of stone disease that may be ubiquitous among all stone formers, regardless of stone type. In addition, data from a larger cohort of mixed calcium stone forming patients, enrolled by convenience sampling for a

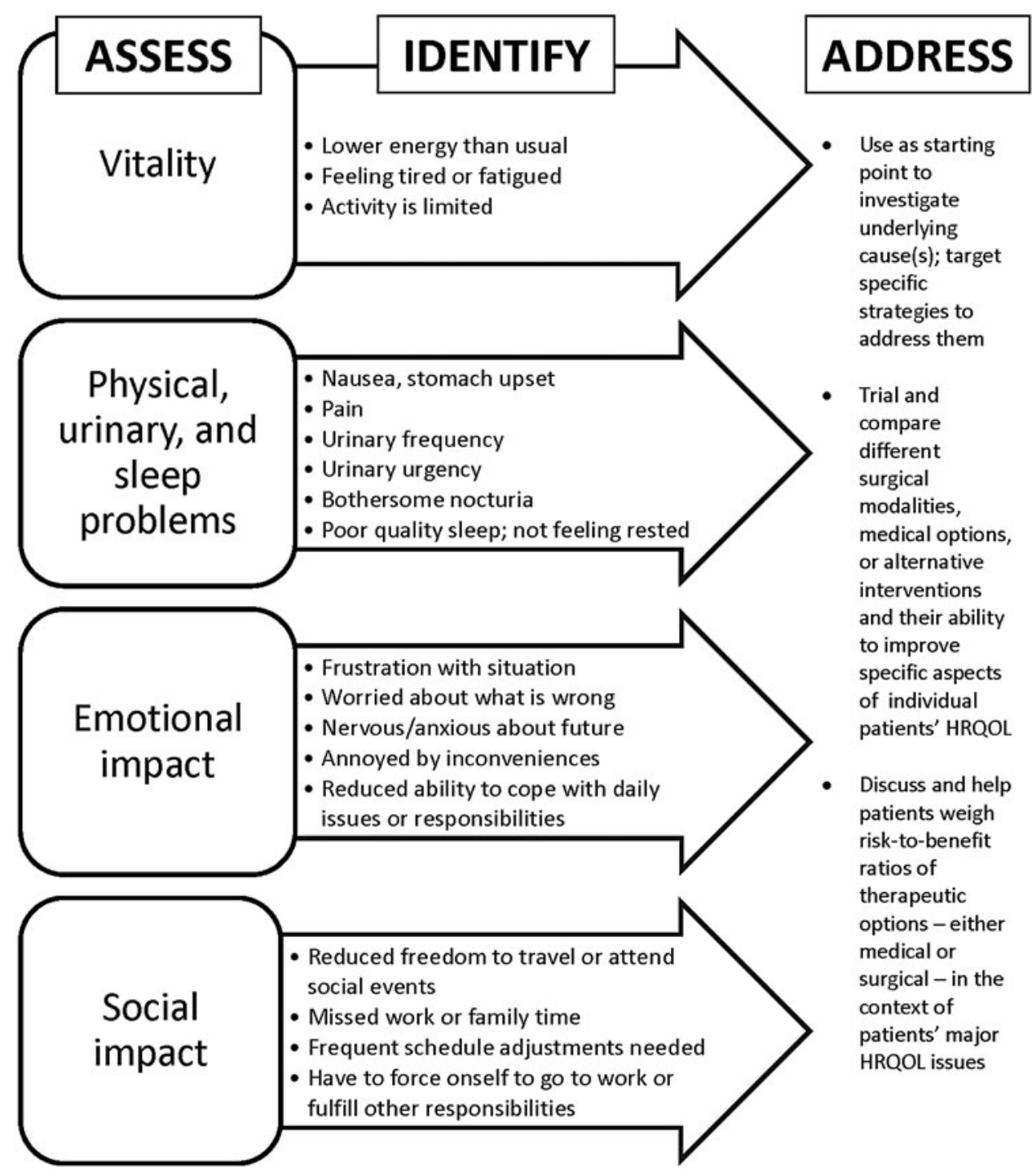

FIG. 1. Assessment and identification of stonespecific influences on healthrelated quality of life may assist in improved disease management. Figure shows subscales of the Wisconsin Stone Quality of Life questionnaire (boxes at left) and some of the specific items within them (arrows), leading toward examples of how disease management may be improved (text at right). 
recent but separate HRQOL study, were used as an additional comparison. The use of this larger group of patients $(n=90)$ as a second control group is consistent with strategies for overcoming sampling bias in case-control studies, especially when the number of cases is low. ${ }^{14}$

Patients in all groups completed the WISQOL, which was either sent by postal mail or given at a clinic appointment. The WISQOL is a 28-item self-administered instrument designed to evaluate the effect of kidney stones on patients' HRQOL. ${ }^{13}$ Items are related to: activity/energy level, sleep patterns, social functioning, therapy compliance, physical symptoms, family life, intimacy, and emotional health; item responses are measured using a 5-point Likert scale. The maximum score for the instrument is $140(28 \times 5)$; a higher score correlates to better HRQOL.

Additional data were obtained from patients' medical records and included: medical history, family history of stones, age of stone onset, number of stone episodes, presence of stone(s) currently, date of last stone episode, number and type of surgical interventions, number of stone passages, history of medical and dietary therapy, presence of current pain/symptoms, and recent emergency room visits due to kidney stones.

Fisher's exact tests were used to evaluate differences in nominal or dichotomous variables. Total WISQOL scores between the cystine group and the other two groups, as well as responses to individual items, were compared with Student's $t$-tests. In addition, subscale scores from four domains identified in a concurrent study involving nine stone centers from the United States and Canada ( $n=1620$ subjects; data not yet published) were compared between groups. In that study, principal component analysis with varimax rotation revealed that four components, incorporating 26 of the 28 WISQOL items, accounted for $>70 \%$ of the variance in WISQOL scores. Items comprising these four components (domains) were thus grouped and summed for the present study as a trial of the discriminatory properties of the subscales. Domains were labeled based on the nature of the items within them and on their patient impact: social impact (domain 1), emotional impact (domain 2), stone-related symptoms or disease impact (domain 3), and vitality (domain 4). A $p$-value $\leq 0.05$ was considered to be significant.

\section{Results}

A total of 52 noncystine recurrent stone forming patients were reviewed with 12 selected as matches. Case-referent matching resulted in both cases and matched controls being $75 \%$ female and with an average of $2.0 \pm 1.6$ comorbidities from a checklist of diseases belonging to the following categories: cardiac, gastrointestinal, pulmonary, endocrine, renal, other genitourinary diseases, blood/circulation, oncology, musculoskeletal, psychiatric, chronic pain, neurologic, and rheumatologic. Mean age was within 3 years (Table 1). Patient variables for the larger group of nonmatched, mixed calcium stone formers were similar for most parameters (Table 1).

\section{Clinical variables}

Patients were similar for time between WISQOL completion and last stone event (mean 1.9, 1.8, and 2.9 years for cystine, matched noncystine controls, and nonmatched, mixed calcium stone forming patients, respectively). Patients with cystine stones had a longer duration of stones (mean 25 vs 16 and 14 years for matched noncystine controls and nonmatched, mixed calcium stone formers, respectively), more lifetime stone episodes $(19 \pm 24$ vs $6.0 \pm 6.1$ and $6.1 \pm 14)$, and a higher frequency of stones $(5.5 \pm 10 v s 0.70 \pm 0.62$ and $0.46 \pm 0.96$ per year). Cystine vs matched noncystine stone formers had a greater number of surgical interventions $(8.5 \pm 9.1 \mathrm{vs}$ $2.9 \pm 3.1$, and surgical intervention modalities were different between these groups with $62 \%$ vs $15 \%$ of cystine $v s$ matched noncystine patients having had percutaneous nephrolithotomy

Table 1. Characteristics of Patients Completing the Wisconsin Stone Quality of Life Questionnaire

\begin{tabular}{|c|c|c|c|c|}
\hline & Cystine & $\begin{array}{c}\text { Matched noncystine } \\
\text { control }\end{array}$ & $\begin{array}{l}\text { Nonmatched } \\
\text { mixed calcium }\end{array}$ & $\mathrm{p}$ \\
\hline Age, year & $50.6 \pm 16.7$ & $53.5 \pm 16.8$ & $54.1 \pm 15.5$ & $0.67 / 0.69$ \\
\hline Family history for stones & $25 \%$ & $33 \%$ & $42 \%$ & $1.0 / 0.35$ \\
\hline Using medication(s) for stone prevention & $50 \%$ & $50 \%$ & $39 \%$ & $1.0 / 0.54$ \\
\hline $\begin{array}{l}\text { Using dietary strategies for stone prevention } \\
\text { (includes hydration) }\end{array}$ & $50 \%$ & $42 \%$ & $67 \%$ & $1.0 / 0.34$ \\
\hline Recurrent stone formers & $100 \%$ & $100 \%$ & $61 \%$ & $1.0 / .007$ \\
\hline \multicolumn{5}{|l|}{ Recent stones } \\
\hline Within 1 year & $67 \%$ & $42 \%$ & $72 \%$ & $0.41 / 0.74$ \\
\hline Within $1-5$ years & $17 \%$ & $58 \%$ & $24 \%$ & $0.09 / 0.73$ \\
\hline More than 5 years ago & $17 \%$ & $0 \%$ & $4 \%$ & $0.48 / 0.15$ \\
\hline Had stones at time of WISQOL completion & $42 \%$ & $42 \%$ & $42 \%$ & $1.0 / 1.0$ \\
\hline $\begin{array}{l}\text { Had stone-related symptoms at time of } \\
\text { WISQOL completion }\end{array}$ & $50 \%$ & $25 \%$ & $18 \%$ & $0.40 / 0.02$ \\
\hline Had sought emergency care within 4 weeks & $8 \%$ & $0 \%$ & $18 \%$ & $1.0 / 0.68$ \\
\hline
\end{tabular}

Data are from (a) patients with cystinuria and a history of cystine stones $(n=12)$, (b) recurrent, mixed calcium stone formers who were matched to patients in the cystine stone cohort for gender, age, and comorbidity $(n=12)$, and (c) a larger cohort of mixed calcium stone formers from our institution $(n=90)$ who were recently enrolled in a larger, institution-wide health-related quality of life study. $p$-Values are from the Student's $t$-test procedure (for age) and from the Fisher's exact test for all other comparisons and represent comparisons between (1) cystine and matched noncystine controls and between (2) cystine and mixed calcium stone formers ( $p$-value $1 / p$-value 2$)$.

WISQOL $=$ Wisconsin Stone Quality of Life questionnaire. 
and $100 \%$ vs $54 \%$ ureteroscopy ( $p \leq 0.04$, Fisher's exact test). The frequency of prior shockwave lithotripsy procedures was equivalent at $15 \%$ for each (numbers and types of stone-removal surgeries for the nonmatched, mixed calcium stone group were not available).

The use of stone-related medications was different between groups. While $50 \%$ of patients in both the cystine and matched noncystine control groups were using stone-related medications, thiazide diuretics were not used in the cystine group but were in $30 \%$ of the matched controls (compared with $15 \%$ of the nonmatched, mixed calcium stone formers). Of patients in the cystine group, 30\% were taking either captopril or Thiola and one also took acetazolamide; no patients in the other two groups used any of these medications. Prescription alkalinizing agents were documented for $30 \%$ of patients in the cystine group, $10 \%$ in the matched noncystine control group, and $28 \%$ in the nonmatched mixed calcium group.

\section{HRQOL scores}

Overall, cystine stone formers had significantly lower total WISQOL scores compared with patients in the other two groups (Table 2). Differences between particular items on the WISQOL were also observed. As a group, cystine patients reported lower scores on items related to sleep problems ( $p \leq 0.050$ for differences between both groups of noncystine patients on 3 WISQOL items), nocturia $(p \leq 0.015)$, and feeling tired or fatigued $(p \leq 0.050)$. In addition, patients in the cystine group tended toward more anxiety about what might go wrong in the future $(p \leq 0.09)$. The comparison of domain scores revealed differences between groups (Table 2).

\section{Patients with vs without stones}

When stratifying our results by patients who reported having stones at the time they completed the WISQOL, cystine patients $(n=5)$ had significantly lower scores than matched noncystine patients $(n=5)$ and also lower than patients in the mixed calcium stone group for WISQOL items related to: having to force oneself to go to work or to fulfill other responsibilities $(p=0.03)$ and for three of six items within the emotional impact domain $(p \leq 0.05)$. Further impact of stones on HRQOL is shown (Table 2).

\section{Discussion}

Using the WISQOL, the only disease-specific instrument for assessing the HRQOL of patients who form kidney stones, we found impaired overall HRQOL in patients who form cystine stones compared with patients with noncystinuric stone disease. Specifically, areas with the lowest HRQOL for cystine patients were those related to sleep problems, nocturia, and feeling tired or fatigued. Cystine patients also had lower scores compared with noncystine patients on items within the emotional impact domain. These items addressed

Table 2. Domain and Total Scores from the Wisconsin Stone Quality of Life Questionnaire Completed by Recurrent Cystine Stone Formers and Mixed Calcium Stone Formers $(N=12$ for Each $)$ and Also by Mixed Calcium Stone Formers $(N=90)$ Who Were Part of a Separate Larger Study

\begin{tabular}{|c|c|c|c|c|}
\hline & Cystine & $\begin{array}{c}\text { Matched noncystine } \\
\text { control }\end{array}$ & $\begin{array}{l}\text { Nonmatched } \\
\text { mixed calcium }\end{array}$ & $\mathrm{p}$ \\
\hline \multicolumn{5}{|c|}{ Domain 1 (social, maximum score, 40$)$} \\
\hline All patients in group & $29.9 \pm 11.2^{\mathrm{a}}$ & $38.0 \pm 3.1$ & $35.0 \pm 7.0$ & 0.045 \\
\hline Patients with stones & $24.0 \pm 12.6^{\mathrm{a}, \mathrm{b}}$ & $38.7 \pm 1.6^{\mathrm{c}}$ & $33.3 \pm 8.7^{\mathrm{a}, \mathrm{d}}$ & 0.067 \\
\hline Patients with no stones & $37.8 \pm 2.6$ & $37.3 \pm 4.8$ & $37.0 \pm 4.9$ & 0.86 \\
\hline \multicolumn{5}{|c|}{ Domain 2 (emotional, maximum score, 35 ) } \\
\hline All patients in group & $23.1 \pm 9.8$ & $30.4 \pm 3.4$ & $27.7 \pm 7.9$ & 0.036 \\
\hline Patients with stones & $15.6 \pm 6.8^{\mathrm{a}, \mathrm{d}}$ & $31.7 \pm 3.0^{\mathrm{a}}$ & $24.7 \pm 9.0^{\mathrm{a}, \mathrm{d}}$ & 0.006 \\
\hline Patients with no stones & $33.5 \pm 3.0$ & $30.5 \pm 4.6$ & $31.1 \pm 4.8$ & 0.32 \\
\hline \multicolumn{5}{|c|}{ Domain 3 (symptoms, maximum score, 40) } \\
\hline All patients in group & $23.7 \pm 10.2^{\mathrm{a}}$ & $32.3 \pm 4.0$ & $31.8 \pm 7.5$ & 0.026 \\
\hline Patients with stones & $20.4 \pm 11.8^{\mathrm{a}}$ & $28.0 \pm 3.8^{\mathrm{d}}$ & $30.1 \pm 8.7^{\mathrm{d}}$ & 0.20 \\
\hline Patients with no stones & $30.8 \pm 6.0$ & $36.0 \pm 1.4$ & $34.2 \pm 5.8$ & 0.14 \\
\hline \multicolumn{5}{|c|}{ Domain 4 (vitality, maximum score, 15 ) } \\
\hline All patients in group & $9.0 \pm 4.8^{\mathrm{a}}$ & $13.0 \pm 2.0$ & $11.4 \pm 3.4$ & 0.028 \\
\hline Patients with stones & $7.6 \pm 5.5^{\mathrm{a}}$ & $11.3 \pm 1.2^{\mathrm{d}}$ & $11.5 \pm 3.8$ & 0.27 \\
\hline Patients with no stones & $12.2 \pm 3.8$ & $14.2 \pm 1.5$ & $11.6 \pm 3.2$ & 0.36 \\
\hline \multicolumn{5}{|c|}{ Total WISQOL score (maximum score, 140) } \\
\hline All patients in group & $93 \pm 37^{\mathrm{a}}$ & $122 \pm 9.6$ & $114 \pm 24$ & 0.021 \\
\hline Patients with stones & $75 \pm 31^{\mathrm{a}, \mathrm{b}}$ & $117 \pm 5.0$ & $108 \pm 29^{\mathrm{d}}$ & 0.061 \\
\hline Patients with no stones & $122 \pm 16$ & $127 \pm 13$ & $122 \pm 17$ & 0.63 \\
\hline
\end{tabular}

$p$-Values are uncorrected values and represent the comparisons between cystine and matched noncystine controls. Other differences are indicated by superscripted numbers. When the Bonferroni correction is applied for the multiple comparisons in the table, only $p$-values $\leq 0.015$ are significant.

${ }^{\mathrm{a}}$ Different than mixed calcium stone formers, $p \leq 0.03$.

${ }^{\mathrm{b}}$ Marginally different within group from patients with no stones, $0.05<p \leq 0.07$.

${ }^{\mathrm{c}}$ Different than mixed calcium stone formers, $0.04 \leq p \leq 0.05$.

${ }^{\mathrm{d}}$ Different within group from patients with no stones, $p \leq 0.015$. 
anxiety or worry about what is wrong or what might go wrong in the future, reduced ability to cope with everyday issues or responsibilities, and irritability. Our findings corroborate those of Modersitzki and colleagues who used the SF-36 questionnaire, a general HRQOL survey, and found cystine stone formers to have lower HRQOL than noncystine stone formers in the category for emotional impact. ${ }^{11}$ In that study, lower HRQOL was also associated with more frequent stone recurrence and with psychiatric comorbidities.

When stratifying within-group results by patients with and without stones at the time they completed the WISQOL, those with stones in all groups scored lower for total score; domain scores for those with stones were also lower (Table 2). However, the magnitude of difference was larger in all cases within the cystine patient group. For all four domains (social, emotional, symptom/disease impact, and vitality), the difference in scores between patients with and without stones for the cystine $v s$ matched noncystine controls and $v s$ the nonmatched, mixed calcium stone forming groups, respectively, was: $14 v s 1$ and 4; 18 vs 1 and 6; 10 vs 8 and 4; and 5 vs 3 and $<1$ (Table 2). For total WISQOL score, cystine patients scored lower than patients in the other groups (47 vs 10 and 14 points lower). While only a few of these differences were statistically significant (Table 2), their clinical relevance appears strong as mathematical differences are obvious. The fact that WISQOL domain scores differed not only between cystine and noncystine groups but also between cystine patients with and without stones suggests that the WISQOL subscales are capable of suitable discrimination. Moreover, the second control group (the 90 nonmatched, mixed calcium stone formers) differed in HRQOL scores from the cystine group to a similar or even greater degree than the matched noncystine controls. This strengthens the finding that the WISQOL will be useful in discriminating unique HRQOL decrements in cystine stone formers.

Compared with the noncystine patients in our study, cystine stone formers had undergone more surgical interventions and more invasive procedures, such as percutaneous nephrolithotomy and ureteroscopy. They also had longer stone disease and more prior stone events. The highly recurrent and aggressive nature of cystine stone disease, as demonstrated by the stone histories of the cystine patients in our study and by others, ${ }^{10,11}$ likely contributes to the impaired HRQOL we observed. Interestingly, one-third of cystine patients had relatively stable disease at the time of WISQOL completion, characterized by their last stone episode having been between 1 and 5 or $>5$ years ago. Nonetheless, their scores were similar to and correlated with cystine patients whose last stone event was more recent. This suggests that cystinuria affects HRQOL even among patients with lower recurrence rates. Further studies are necessary to evaluate whether changes in HRQOL are possible with improved preventive and/or disease management strategies.

The effectiveness of stone management has historically been assessed with objective outcomes such as stone-free status and recurrence. However, HRQOL is recognized as an important measure in healthcare, and treatment decisions are increasingly expected to take into account the psychosocial, physical, and economic impact associated with disease. Several studies have previously assessed HRQOL in patients who form kidney stones ${ }^{10,11,13-20}$ and also in those who have undergone surgical stone removal procedures. ${ }^{21-24}$ Cystine stone formers represent a unique population with particularly aggressive disease that can be difficult to manage. Our study demonstrates particular HRQOL impairments in this population. Compliance with preventive medical regimens may not be optimal in all patients. Pareek and colleagues found that cystine patients who were not compliant with medical and nutritional therapy had a higher need for surgical intervention. ${ }^{5}$ While our study did not compare the HRQOL of compliant patients with those who were not, it is possible that those with suboptimal compliance with prevention suffer more and have lower HRQOL. In contrast, the impact on HRQOL of compliance with medical management is unknown.

Chronic diseases and their related impacts on activity, personal finances, and emotional and social functioning are a major health problem; studies link many chronic diseases with lower HRQOL. ${ }^{25}$ Self-perceptions of health predict mortality rate, morbidity, and future use of healthcare services, underscoring the value of tracking the burden of disease in our patients and prompting the development of programs and regimens to address HRQOL deficits. Understanding the effects of cystinuria and stones on patients' HRQOL may lead to improvements in disease management. Identification of a patient's specific HRQOL impairments could be a starting point for a discussion about underlying contributors, which could then be addressed. For example, patients' lower scores on items related to emotional impact could be due to fear or anxiety about an impending invasive surgical procedure. In this event, a less invasive staged procedure may be offered. In another example, the identification of contributors to lower HRQOL related to nocturia and/or other sleep problems could lead to adjustments in fluid recommendations and/or to a referral to a sleep specialist. Studies evaluating the effect of tailored disease management on patients' stone-related HRQOL would be helpful.

Limitations of this study include a small population with low response rate from cystine patients; however, cystine stones are rare. In addition, this is a single institution study, and there may be variations in a more diverse population. Furthermore, our results describe patients from a tertiary center and may not be generalized to patients treated in the community. In this small study, we were unable to assess compliance with medication and/or dietary therapy and its impact on HRQOL. Finally, the clinical significance of the differences observed using the WISQOL is unknown as this has not yet been assessed, but is underway in our ongoing psychometric analyses of this instrument.

\section{Conclusions}

Through the use of a disease-specific quality of life instrument (WISQOL), ${ }^{13}$ we identified unique symptoms and psychosocial challenges associated with patients who form cystine stones, revealing areas in which they may fare even worse than other stone-forming patients. Specific areas of decrement included sleep problems, nocturia, and feeling tired or fatigued. Items related to the emotional impact of stones also appeared to be more bothersome among cystine $v s$ noncystine stone formers. By identifying areas of decreased HRQOL, specific disease management and coping skills may be developed and tailored to patients who form cystine stones. 


\section{Author Disclosure Statement}

No competing financial interests exist.

\section{References}

1. Richet G. The chemistry of urinary stones around 1800: A first in clinical chemistry. Kidney Int 1995;48:876-886.

2. Sakhaee K. Pathogenesis and medical management of cystinuria. Semin Nephrol 1996;16:435-447.

3. Chow GK, Streem SB. Medical treatment of cystinuria: Results of contemporary clinical practice. J Urol 1996;156: 1576-1578.

4. Castro Pereira DJ, Schoolwerth A, Pais VM. Cystinuria: Current concepts and future directions. Clin Nephrol 2015;83:138-146.

5. Pareek G, Steele TH, Nakada SY. Urological intervention in patients with cystinuria is decreased with medical compliance. J Urol 2005;174:2250-2252.

6. Assimos DG, Leslie SW, Ng C, et al. The impact of cystinuria on renal function. J Urol 2002;168:27-30.

7. Lindell A, Denneberg T, Granerus G. Studies on renal function in patients with cystinuria. Nephron 1997;77:76-85.

8. Pietrow P, Auge BK, Weizer AZ, et al. Durability of the medical management of cystinuria. J Urol 2003;169:68-70.

9. Parks JH, Asplin JR, Coe FL. Patient adherence to longterm medical treatment of kidney stones. J Urol 2001;166: 2057-2060.

10. Parr JM, Desai D, Winkle D. Natural history and quality of life in patients with cystine urolithiasis: A single centre study. BJU Int 2015;116:(Suppl 3)31-35.

11. Modersitzki F, Pizzi L, Grasso M, et al. Health-related quality of life (HRQoL) in cystine compared to non-cystine stone formers. Urolithiasis 2014;42:53-60.

12. Bergman J, Gore JL, Singer JS, et al. Readability of health related quality of life instruments in urology. J Urol 2010; 183:1977-1981.

13. Penniston KL, Nakada SY. Development of an instrument to assess the health related quality of life of kidney stone formers. J Urol 2013;189:921-930.

14. Mann CJ. Observational research methods: Research design II-cohort, cross sectional, and case-control studies. Emerg Med J 2003;20:54-60.

15. Penniston KL, Nakada SY. Health related quality of life differs between male and female stone forms. J Urol 2007; 178:2435-2440.

16. Diniz DH, Blay SL, Schor N. Anxiety and depression symptoms in recurrent painful renal lithiasis colic. Braz $\mathrm{J}$ Med Biol Res 2007;40:949-955.
17. Bensalah K, Tuncel A, Gupta A, et al: Determinants of quality of life for patients with kidney stones. J Urol 2008; 179:2238-2243.

18. Donnally CJ 3rd, Gupta A, Bensalah K, et al: Longitudinal evaluation of the SF-36 quality of life questionnaire in patients with kidney stones. Urol Res 2001;39:141-146.

19. Bryant M, Angell J, Tu H, et al: Health related quality of life for stone formers. J Urol 2012;188:436-440.

20. Angell J, Bryant M, Tu H, et al: Association of depression and urolithiasis. Urology 2012;79:518-525.

21. Damiano R, Autorino R, De Sio M, et al. Does the size of ureteral stent impact urinary symptoms and quality of life? A prospective randomized study. Eur Urol 2005;48: 673-678.

22. Joshi HB, Stainthorpe A, MacDonagh KP, et al. Indwelling ureteral stents: Evaluation of symptoms, quality of life and utility. J Urol 2003;169:1065-1069.

23. Kouriefs C, Quatan N, Larking A, et al. What is important to patients receiving extracorporeal shock-wave lithotripsy for urinary tract calculi? Ann R Coll Surg Engl 2004;86: 432-435.

24. Mays NB, Petruckevitch A, Snowdon C. Patients' quality of life following extracorporeal shock-wave lithotripsy and percutaneous nephrolithotomy for renal calculi. Int $\mathbf{J}$ Technol Assess Health Care 1990;6:633-642.

25. Campbell VA, Crews JE, Moriarty DG, et al. Surveillance for sensory impairment, activity limitation, and healthrelated quality of life among older adults: United States, 1993-1997. MMWR CDC Surveill Summ 1999;48:131156.

Address correspondence to: Kristina L. Penniston, PhD, RD Department of Urology

University of Wisconsin School of Medicine and Public Health 1685 Highland Avenue $3258 \mathrm{MFCB}$ Madison, WI 53705-2281

E-mail: penn@urology.wisc.edu

Abbreviations Used
HRQOL $=$ health-related quality of life
WISQOL $=$ Wisconsin Stone Quality of Life questionnaire

